\title{
Seroprevalence of Toxoplasma gondii infection in poultry kept under different housing conditions in Israel
}

Salant, H. ${ }^{1 *}$, Hamburger, J. ${ }^{1}$, Spira, D. ${ }^{1}$, Ben David, A. ${ }^{2}$, Schwan, E.V. ${ }^{3}$

${ }^{1}$ Department of Microbiology and Molecular Genetics, The Kuvin Center for the Study of Infectious and Tropical Diseases, Hadassah Medical School, Hebrew University, Jerusalem, Israel.

${ }^{2}$ Self Employed Field Poultry Veterinarian, 15 Kipodan St Modi'in, Israel.

${ }^{3}$ Department of Veterinary Tropical Diseases, Faculty of Veterinary Science, University of Pretoria, Private Bag X04, Onderstepoort 0110, South Africa.

*Corresponding author: H. Salant, Department of Microbiology and Molecular Genetics, The Kuvin Center for the Study of Infectious and Tropical Diseases, Hadassah Medical School, Hebrew University, POB 12272, Jerusalem 91120, Israel; Tel.: ++972 2 675-8080; Fax:++97226757425; Email: tsvis@ekmd.huji.ac.il 
Abstract

A Toxoplasma gondii seroprevalence survey was conducted in poultry flocks kept under different housing systems in Israel. The seroprevalence rates were $35.4 \%$ (46/130) in battery-raised chicken egg-layers from Jerusalem which were first raised on the ground, $12.5 \%(19 / 152)$ in free-range chickens from various locations of the country and $9 \%$ (4/45) in turkeys from the north. However, all broilers (50) and chicken breeding stock (58) kept under high biosecurity conditions in Jerusalem were found to be $T$. gondii seronegative. It is concluded that high biosecurity conditions prevent contamination with oocysts by rendering premises inaccessible to cats.

Keywords: Toxoplasma gondii, poultry, housing methods, seroprevalence, MAT 


\section{Introduction}

Toxoplasmosis is caused by Toxoplasma gondii, a coccidian intracellular protozoan parasite. The domestic cat and to a lesser extent wild felids act as definitive hosts while several mammal and bird species act as intermediate hosts. Toxoplasmosis is one of the most common parasitic zoonosis and it is estimated that a third of the world population is seropositive (Saadatnia and Golkar, 2012). Humans can contract toxoplasmosis by ingesting sporulated oocysts originating from cat faeces, by consumption of tissue cysts in raw infected meat or transplacentally in primary infected women during pregnancy (Dubey and Lindsay, 2004; Innes, 1997). In immune competent humans, toxoplasmosis is in general clinically uneventful (Tenter et al., 2000). However, severe pathological effects can result in immunocompromised individuals as well as in fetuses and neonates following primary infections of women, contracted during pregnancy (Dubey and Jones, 2008).

Chickens are clinically resistant to $T$. gondii, but play a significant role in the epidemiology of toxoplasmosis in the rural environment and are an efficient source of infection for cats (Dubey, 2002; Dubey, 2010). Sources of infection for poultry are primarily feed contaminated with sporulated oocysts from the environment and probably to a lesser extent invertebrates (earthworms, molluscs, cockroaches, flies, beetles) acting as transport hosts for oocysts (Dubey, 2002; Dumètre and Dardè, 2003; Ruiz and Frenkel, 1980). A previous survey in the northern part of Israel found a high infection rate in a commercially raised flock of chicken egg-layers with $42.2 \%$ of seropositive birds harbouring viable parasites (Dubey et al., 2004). Free-range 
chickens are invariably exposed to oocysts shed by cats. Also domestic turkeys can act as intermediate hosts but, similar to chickens, are resistant to clinical toxoplasmosis (Dubey et al., 1993a; Sedlák et al., 2000).

Since Israel has the world's largest per capita consumption of turkey meat and the fourth largest per capita consumption of chicken meat (http://www.thepoultrysite.com/), it warrants the need to obtain data on seroprevalence in poultry flocks, particularly with regard to different housing systems.

Published information regarding Toxoplasma seroprevalence in poultry kept in different housing systems is very limited. A survey conducted in northeast China reported significant differences in seroprevalence rates in cage-raised poultry $(2.8 \%)$ compared to free-range poultry (34.7\%) (Zhu et al., 2008). In another survey conducted in southern China, Toxoplasma antibody titers were found in $16 \%$ of ducks, $11.4 \%$ of free-range chickens and $4.1 \%$ of caged chickens sampled (Yan et al., 2009). Serological surveys conducted in geese and ducks in Germany revealed that flocks kept exclusively indoors had a significantly lower number of positive birds when compared to flocks kept outdoors (Maksimov et al., 2011).

The objective of the present survey was to compare $T$. gondii seroprevalence rates of poultry flocks kept under different housing systems in Israel. The hypothesis is that the extent of exposure to sporulated oocysts depends largely on the housing system, with systems in which birds are raised above the ground and (or) overall high biosecurity providing significantly more protection from infection. 
2. Materials and Methods

\subsection{Survey area}

The survey was conducted on poultry flocks from the Jerusalem, North of Tel-Aviv and Samaria districts in Israel.

\subsection{Study animals}

Blood samples from a total of 390 chickens and 45 turkeys were obtained for the survey. Chickens (Gallus domesticus) included in the survey belonged to several groups with respect to their housing conditions. Group 1a consisted of 100 21/2-yearold commercially raised free-range egg-layers raised in Shilo, in the central part of Israel, approximately $45 \mathrm{~km}$ northeast of Tel-Aviv. Group $\mathbf{1 b}$ consisted of 52 household free-range chickens up to 11/2-year-old from the mainly Arab inhabited area of East Jerusalem. Group 2 comprised 130 battery-raised egg-laying chickens ranging between 14-127 weeks of age, from 6 different flocks in the Jerusalem area. They were housed on the ground until the age of 105 days and subsequently above the ground in cages. The battery raised chickens were divided into those that had an average age of 29 weeks ( 3 flocks) and those older birds with an average age of 110 weeks (3 flocks). Chickens in all groups $(1 \mathrm{a}, 1 \mathrm{~b}, 2)$ were likely to be exposed to $T$. gondii oocysts shed by cats. Group 3 consisted of 50 broilers kept on concrete floors until 6 weeks of age. Chickens in Group 4 comprised 58 breeders which were over 2 years of age at sampling. Both, groups 3 and 4, were from the Jerusalem area and raised under strict biosecurity. Group 5 comprised 45 turkeys (Meleagris gallopavo) 
used for their meat from a farm situated approximately $25 \mathrm{~km}$ north of Tel-Aviv. They were exposed to feral cats. Their ages were approximately 5 months at the time of sampling. Samples from groups 1 and 5 were collected in September 2004 whilst samples from other groups were collected from the beginning of July to the end of August 2008.

\subsection{Blood samples}

Poultry of Groups 1a and Group 5 were destined for slaughter and the latter mentioned group for subsequent human consumption. Blood samples were collected individually into serum collection tubes during the slaughtering process at Atarot 'Halal' Slaughterhouse in Jerusalem. Blood samples were centrifuged at $3000 \mathrm{~g}$ for 15 min and the sera stored at $-20{ }^{0} \mathrm{C}$ until screened. Serum samples from poultry in Groups 1b, 2, 3, 4 were provided by a coauthor (ABD) collected for routine disease screening.

\subsection{Serological assay}

Serological testing for $T$. gondii $\mathrm{IgG}$ antibodies was performed using the modified agglutination test (MAT) (Dubey and Desmonts, 1987). Compared to other serological tests, the MAT has a high sensitivity and specificity in domestic animals (Dubey et al., 1993b; Dubey, 1997, Shaapan et al., 2008; Casartelli-Alves et al.; 2014). The MAT detects only IgG antibodies as mercaptoethanol used in the test destroys IgM antibodies (Dubey, 2010). 
Formalinized T. gondii tachyzoites in phosphate- buffered saline as antigen for the MAT were provided by the Laboratoire de la Toxoplasmose, Paris, France. Positive and negative sera from poultry were provided by the United States Department of Agriculture in Beltsville, USA. Serum samples from chickens infected and confirmed to be Eimeria tenella positive by the Israel Poultry Board were provided by coauthor, ABD to exclude cross-reactivity of this species with $T$. gondii in the MAT. Sera from all poultry were diluted two-fold from a baseline titer of 1:5 which was regarded as positive.

\subsection{Statistical analysis}

The SPSS16 (SPSS Inc, Chicago, Illinois, USA) computer package was used for analysis of data. Data were also analyzed by using Fisher's exact test for categorical variables. Values of $p<0.05$ were taken as significant. 


\section{Results}

Serum samples from a total of 390 chickens and 45 turkeys were screened for the presence of $T$. gondii antibodies. The seroprevalence in chickens in Groups 1a and $1 \mathrm{~b}$ (free-range egg layers) was $12.5 \%$ (19/152). Forty-six of $130(35.4 \%)$ battery-raised egg layers (Group 2) were found to be $T$. gondii seropositive; 0/50 (0\%) of 6-weekold broilers (Group 3) were seropositive; and 0/58 (0\%) of chickens used for breeding stock (Group 4) were seropositive. Four of 45 (8.9 \%) 5 month-old commercially raised turkeys (Group 5) were seropositive (Figure 1). The average $T$. gondii seroprevalence of 3 battery raised egg laying flocks 29 weeks of age was found to be $39.5 \%$ compared to an average $T$. gondii seroprevalence of $60.5 \%$ in three flocks 110 weeks of age (Results not shown). Although it had been hypothesized and, in fact was observed that the sum of groups, battery-raised chickens (Group 2), broilers (Group 3) and breeding stock (Group 4), with a total of 46 positive birds out of $238(19.3 \%)$, was significantly more $T$. gondii seropositive than the sum of freerange chickens (Groups 1a and 1b) and turkeys (Group 5), with a total of 23 positive birds out of $197(11.6 \%, \mathrm{p}=0.02)$, the observation of a specifically high seroprevalence rate among battery-raised chickens (Group 2), (46/130; $35.4 \%)$ contrary to the initial hypothesis' expectation necessitated this group to be compared to the sum of all other groups $(23 / 305 ; 7.5 \%)$. The difference was found to be of very high significance $\left(\mathrm{p}=4.24 \times 10^{-12}\right)$. Thus, this group was removed for comparison with other groups in the analysis. The sum of $T$. gondii seroprevalence amongst broilers (Group 3) and chicken breeding stock (Group 4) with a total of 0/108 (0\%) was significantly different $\left(\mathrm{p}=2.65 \times 10^{-5}\right)$ compared to the sum of seroprevalence of free range chickens (Groups 1a and 1b) and turkeys (Group 5), with a total of 23/197 
(11.7\%). Similar to findings obtained by Dubey and others (1993) this survey demonstrated no cross-reaction in the MAT with sera obtained from Eimeria tenellainfected chickens used as controls. 


\section{Discussion}

The survey demonstrated that poultry is widely exposed to $T$. gondii in Israel (Fig. 1). Importantly, and as has been mentioned in the results section, this MAT assay demonstrated high specificity as no cross-reaction was observed with sera obtained from Eimeria tenella-infected chickens used as controls.

The results of the survey present evidence that housing conditions which prevent oocyst-shedding cats to access premises have a significant impact on $T$. gondii seroprevalence of poultry (Fig. 1). Battery-raised egg layers (Group 2) had the highest cumulative seroprevalence of all 6 survey groups with $35.4 \%$ of birds infected (Fig 3.1). Housing egg-laying chickens in batteries above the ground did not change the level of $T$. gondii seroprevalence since they were initially housed on the ground until the age of 105 days. A varying lack of strict biosecurity conditions in this group, which was personally observed, with rodents and cats occasionally gaining access to the housing facilities might be a possible explanation. It might also be related to differences in the age composition of these groups. Conditions of high bird concentrations in limited spaces where cats readily frequent may allow for easier $T$. gondii oocyst exposure allowing for these high seroprevalence rates to be achieved. Broilers in Israel are often raised on cement pens and on the ground under conditions of relatively high biosecurity with regard to access to cats and rodents. Broiler raising pens are thoroughly cleaned and disinfected with aldehydes after each batch of broilers is removed and before reintroduction of a new flock. This is probably also the reason why birds in Group 3 were seronegative. Even the relatively much older 2year-old breeders (Group 4) were found to be seronegative and this can only be explained by an effective housing system where biosecurity is strictly enforced and 
feed/litter is not contaminated with oocysts. Zhu et al., (2008), Yan et al., (2009) and Maksimov et al., (2011) have reported similar findings amongst biosecurity-enforced, caged and free-range poultry in North East China and enclosed and free-range geese and ducks in Saxony, Germany. Both reports relate high $T$. gondii seroprevalence in free-range birds due to their exposure to feral cats. Unfortunately, these surveys compared only two types of housed poultry and did not include the possibility of housing birds above the ground exclusively in their investigations.

Although relatively few turkeys were sampled in the survey, close to 10 percent of those birds sampled (4/45) were seropositive for T. gondii anntibodies. In fact, three of 4 birds had antibody titers that were $\geq 50$ (Results not shown). This finding should signal a warning to stakeholders in the poultry industry and officials involved in public health. Further surveys should be conducted in turkeys using larger sample groups in order to confirm the high seroprevalence of $T$. gondii in this poultry species since turkey meat is consumed extensively by the Israeli public (http://www.thepoultrysite.com/).

Housing conditions as a modifying mechanism for $T$. gondii prevalence in poultry is crucial, especially in a day and age whereby animal production systems that offer outdoor access to animals have become increasingly popular in the Western world, including Israel, due to the growing general discontent of consumers with conventional bio-industrial farming practices. These open production systems offer improved animal welfare but may create new problems for animal health, resulting in increased food safety risks from parasitic infections, like toxoplasmosis. In current organic husbandry systems, the animals are kept at lower stocking densities and have outdoor access. The design of new animal production systems with outdoor access 
requires both a thorough analysis of possible risks and optimal communication of these risks throughout the food chain and appropriate partitioning of responsibility concerning these risks. Some risks are inherent to the choice of keeping animals in a more natural environment and could be judged as an inherent responsibility of the consumer, whereas other risks may be mitigated by further refinement or adjustment of the housing or farm management system used.

In conclusion, it is important to adopt strategies to house poultry according to the ability to reduce $T$. gondii exposure from infective cats and other infected animals with subsequent infection in birds using effective affordable biosecure based housing.

\section{Acknowledgements}

We would like to thank Dr. J.P. Dubey of the USDA ,Beltsville MD, USA for furnishing of positive and negative serum samples as internal controls for the assay and Prof. Norman Grover of Hadassah Medical School, Hebrew University of Jerusalem for assisting with the statistical analysis of the results.

6. Competing interests

This research did not receive any specific grant from funding agencies in the public, commercial, or non-for-profit sectors. 


\section{References}

Casartelli-Alves, L., Boechat, V.C. Macedo-Couto, R., Ferreira, L.C., Nicolau, J.L., Neves, L.B., Millar, P.R., Vicente, R.T., Oliveira, R.V., Muniz, A.G., Bonna, I.C., Amendoeira, M.R., Silva, R.C., Langoni, H., Schubach, T.M., Menezes, R.C., 2014. Sensitivity and specificity of serological tests, histopathology and immunohistochemistry for detection of Toxoplasma gondii infection in domestic chickens. Vet. Parasitol. 204, 346-351.

Dubey, J.P., Desmonts, G., 1987. Serological responses of equids fed Toxoplasma gondii oocysts. Equine Vet. J. 19, 337-339.

Dubey, J.P., Thulliez, P., 1993. Persistence of tissue cysts in edible tissues of cattle fed Toxoplasma gondii oocysts. Am. J. Vet. Res. 54, 270-273.

Dubey, J.P., Camargo, M.E., Ruff, M.D., Wilkins, G.D., Shen, S.K., Kwok, O.C.H., Thulliez, P., 1993a. Experimental toxoplasmosis in turkeys. J. Parasitol. 79, 949-952.

Dubey, J.P., Ruff, M.D., Camargo, M.E., Shen, S.K., Wilkins, G.L., Kwok, O.C.H., Thulliez, P., 1993b. Serologic and parasitologic responses of domestic chickens after oral inoculation with Toxoplasma gondii oocysts. Am. J. Vet. Res. 54, 1668-1672.

Dubey, J.P., 1997. Validation of the specificity of the modified agglutination test for toxoplasmosis in pigs. Vet. Parasitol. 71, 307-310. 
Dubey, J.P., 2002. A review of toxoplasmosis in wild birds. Vet. Parasitol. 106, 121153.

Dubey, J.P., Salant, H., Sreekumar, C., Dahl, E., Vianna, M.C., Shen, S.K., Kwok, O.C., Spira, D., Hamburger, J., Lehmann, T.V., 2004. High prevalence of Toxoplasma gondii in a commercial flock of chickens in Israel, and public health implications of free-range farming. Vet. Parasitol. 121, 317-322.

Dubey, J.P., Lindsay, D.S., 2004, 'Biology of Toxoplasma gondii in cats and other animals. World Class Parasites, Volume 9. Opportunistic Infections: Toxoplasma, Sarcocystis, and Microsporidia. Springer, US.

Dubey, J.P., Jones, J.L., 2008. Toxoplasma gondii infection in humans and animals in the United States. Int. J. Parasitol. 38, 1257-1278.

Dubey, J.P., 2010. Toxoplasma gondii infections in chickens (Gallus domesticus): prevalence, clinical disease, diagnosis and public health significance. Zoonoses Public Hlth. 57(1), 60-73.

Dumètre, A. \& Dardè, M.L., 2003. How to detect Toxoplasma gondii oocycts in environmental samples? FEMS Microbiol. Rev. 27, 651-661.

European Council. 2007. Council Regulation (3C) No 834/2007 of 28 June 2007 on organic production and labeling of organic products and repealing Regulation (EEC) No 2092/91. Official Journal of the European Union- Lex. 189, 1-23. 
http://www.thepoultrysite.com/

Innes, E.A., 1997. Toxoplasmosis: comparative species susceptibility and host immune response. Comp. Immunol., Microbiol. Infect. Dis. 20, 131-138.

Maksimov, P., Buschtöns, S., Herrmann, D.C., Conraths, F.J., Görlich, K., Tenter, A.M., Dubey, J.P., Nagel-Kohl, U., Thoms, B., Bötcher, L., Kühne, M., Schares, G., 2011. Serological survey and risk factors for Toxoplasma gondii in domestic ducks and geese in Lower Saxony, Germany. Vet. Parasitol. 18(2-4), 140-149.

Ruiz, A. \& Frenkel, J.K., 1980. Intermediate and transport hosts of Toxoplasma gondii in Costa Rica. Am. J. Trop. Med. Hyg. 29, 1161-1166.

Saadatnia, G. \& Golkar, M., 2012. A review on human toxoplasmosis. Scand. J. Infect. Dis. 44, 805-814.

Sedlák, K., Literák, I., Vitula, F. \& Benák, J., 2000. High susceptibility of partridges (Perdix perdix) to toxoplasmosis compared with other gallinaceous birds. Avian Pathol. 29, 663-569.

Shaapan, R.M., El-Nawawi, F.A., Tawfik, M.A., 2008.Sensitivity and specificity of various serological tests for the detection of Toxoplasma gondii infection in naturally infected sheep. Vet. Parasitol. 153, 359-362. 
Tenter, A.M., Heckeroth, A.R. \& Weiss, L.M., 2000. Toxoplasma gondii: from animals to humans. Int. J. Parasitol. 30, 1217-1258.

Yan, C., Yue, C.L., Yuan, Z.G., He, Y., Yin, C.C., Lin, R.Q., Dubey, J.P., Zhu , X.Q., 2009. Toxoplasma gondii infection in domestic ducks, free-range and caged chickens in southern China. Vet. Parasitol. 165, 337-340.

Zhu, J., Yin, J., Xiao, Y., Jiang, N., Andale, J., Lind, J. \& Chen, Q., 2008. A seroepidemiological survey of Toxoplasma gondii infection in free-range and caged chickens in northeast China. Vet. Parasitol. 158, 360-363. 
Figure 1: Toxoplasma gondii seroprevalence in poultry kept under different housing conditions in Israel 


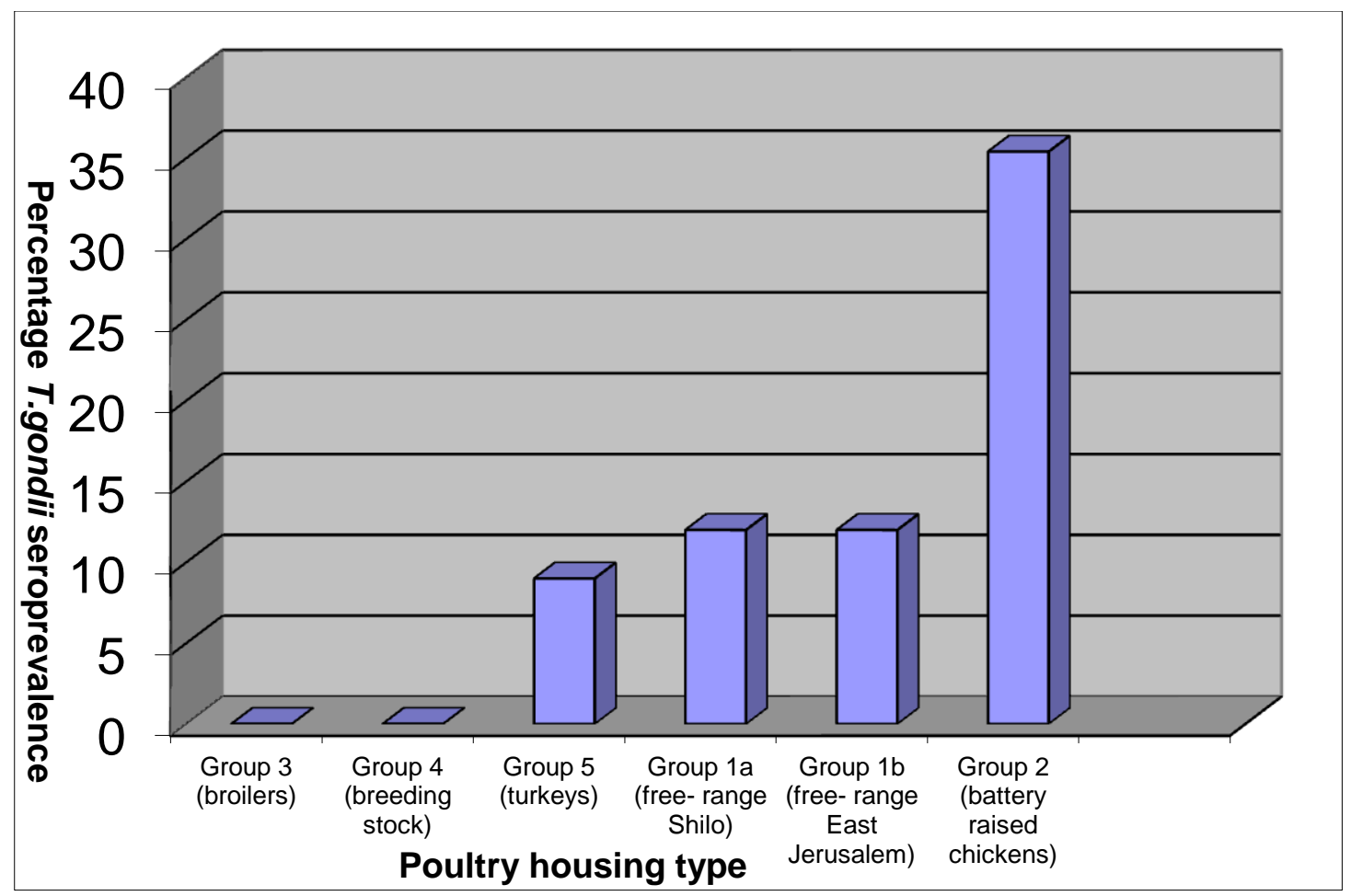

